Widefield ScIENCE AND TeChNOLOGY For the SKA

SKADS CONFERENCE 2009

S.A. Torchinsky, A. van Ardenne, T. van den Brink-Havinga, A.J.J. van Es, A.J. Faulkner (eds.)

4-6 November 2009, Château de Limelette, Belgium

\title{
AAVP: The Next Step after SKADS
}

\author{
A. van Ardenne $e^{1,2}$ \\ 1 ASTRON - Oude Hoogeveensedijk 4, 7991 PD Dwingeloo, The Netherlands \\ ${ }^{2}$ Chalmers University of Technology- SE-412 96 Gothenburg, Sweden
}

\begin{abstract}
An introductory overview of the Aperture Array Verification Programme (AAVP), as follow-up program from SKADS is given.AAVP will be continuing to strengthen European collaborations as in SKADSdeveloping the aperture arrays into an SKA ready instrument. It will focus on the design and development of both AA-low (from 70-450 MHz) and onAA-high (from 400-1400 MHz) as averification system demonstratingdeep imaging among others. This European led3-years program will be executed in close cooperation with the SPDO by 15 institutes in 9 European Countries with active participation from Australia and South Africa.
\end{abstract}

\section{Introduction}

Many of the key science drivers for the SKA - such as moderate to high redshift HI surveys for Galaxy formation and evolution, studies of Baryonic Oscillations, searches for (ms-) pulsars and transients as well as cosmic magnetism - depend on a large instantaneous field of view, FoV, at mid-SKA frequencies for the very high survey speeds required.

Europe is expecting to contribute significantly to the funding of the SKA construction and is very well positioned to play a major role in developing SKA wide-field survey capability. The basis of the aperture array technologies is from LOFAR and SKADS plus phased focal plane arrays with APERTIF. The large FoV of aperture arrays is a key performance indicator in the search for transients (whether Cosmological, Extragalactic, Galactic or ET's). However, in addition to the FoV, the extremely short time to start observing in any direction is a second key asset of aperture arrays. Indeed the speed of aperture arrays for locking onto a new celestial radio source may well be crucial; reactions will have to be within seconds to external (e.g. robotic optical telescopes or orbiting satellites) or internal SKA triggers.

SKADS has produced science simulations, costed engineering and technology development, completed in December 2009. This work will be continued and enhanced by its successor the Aperture Array Verification Program, AAVP.

The AAVP is the next step in the development of An SKA Aperture Array system operating from 0.07- 1.4 GHz.

This system will be implemented by using a sparse AA-Lo array up to about $400 \mathrm{MHz}$ or higher, to counter the high sky noise temperatures at low frequencies and using the experience from current arrays, principally LOFAR \& MWA, but with a much higher sensitivity, up to factor of 100x. Frequencies up to about $1.4 \mathrm{GHz}$, will use a dense Aperture Array, AA-hi, researched in SKADS, to provide the very high survey speed required by the key SKA science. Both arrays sample the sky directly i.e. without any concentrator such as dishes as described in full detail in these Proceedings.

More background considering the development of widefield surveying capability is discussed in SKA Memo 108
"Determining the Specification of an Aperture Array for Cosmological Surveys".

Although the mid-frequency SKA scientific applications described above need a wide FoV, it is obvious that nearly all SKA science drivers will benefit from a larger FoV. So the real question is not whether we need it - 'yes we do' - but whether we can afford a wide FoV in time, cost and proven performance. The essence of this conclusion can be formulated into two further questions:

- In the overall cost equation for the mid-frequency SKA, what are the cost comparisons in both hardware and processing for the AA solution compared to the dish solution for a given $A_{\text {eff }} / T_{\text {sys }}$ ?

- Can we show that the performance, in terms of achievable dynamic range, of aperture arrays is not inherently worse than that of dishes, with or without FPA's? Indeed could AA's actually be better since we are dealing with large unblocked apertures and there is exquisite control over the "surface"?

Answers to the second question can be obtained through a well-planned testing and simulation program in which the AAVP will play the driving role.

Recent observations with the WSRT have shown that a central issue for all collectors including aperture arrays is likely to be the spectral dynamic range, in particular the off-axis spectral behaviour. For the WSRT they are known as the ' $17 \mathrm{MHz}$ ripple' (e.g. Popping \& Braun, 2008). The current spectral dynamic range achieved on-axis is typically $10^{3}-10^{4}$ in the WSRT and at least $10 \times$ worse off-axis; for SKA this range will have to be at least $10^{6}$. The required performance for continuum observations will have to be improved by a factor of $\sim 100$, note that broad continuum bands are typically built up from $\sim 10,000$ narrow line channels, hence there will be the same spectral dynamic range limitations.

The AAVP program is a three year programmatic collaboration of institutes in 9 European countries, Australia and South Africa. It aims to advance AA's to the level that a costed engineering and fully verified proven design can be developed as input to the production engineering for SKA Phase 1. The pro- 
gram is structured such that activities are well coordinated with PrepSKA and the SKA Program Development Office ensuring optimal contribution to the SKA timescale and specification goals.

To implement the AAVP, the European SKA Consortium (ESKAC) has established an AAVP group which will coordinate the technical developments and testing. As with SKADS, ESKAC represents the role and extensive interests, visions and ambitions of the European astronomical science and engineering community in the SKA, acting as the umbrella organization for AAVP.

The AAVP work will be carried out in agreement and collaboration with the SPDO. The AAVP will in many respects continue the role of SKADS, while not being a funded European project with the consequent formal financial reporting structure; the AAVP will actively align and report on the development work for the aperture arrays in a structured fashion and provide a forum for European technical exchange.

As with the Dish Verification Program (DVP), AAVP is now also part of the EC-FP7 PrepSKA global effort in defining and organizing the SKA.

With this background, this paper further details the organization and objectives of the AAVP.

\section{Scope}

Activities in SKADS have identified the key outstanding issues to be addressed through the AAVP.

These relate to (i) maturing preferred technologies and making selections, (ii) making architectural and design choices for Phase 1 and Phase 2 SKA, and (iii) demonstrating the performance of AA's for SKA radio astronomy in terms of performance, power, cost and timeline.

Therefore, the AAVP will provide the following:

- Coordination of the European technical work, primarily for aperture arrays.

- Ensure that the verification programme for aperture arrays endeavors to meet the requirements of the SKA Project and ongoing assessments of concept affordability/feasibility along the lines mentioned above.

- A focus and information exchange for European SKA development work e.g. by organizing European technical meetings and conferences.

- Deliverables on a timescale commensurate with PrepSKA/SKA

Participation is from 15 institutes over 9 European Countries plus Australia and South Africa as associated countries. The program overall when fully funded from national resources is expected to be valued at the level of about $18 \mathrm{M} €$ and will last until the end of 2012. The funded contribution is expected to cover the required manpower of approximately 3540 FTE per year over the 3 years and the associated materials, traveling etc.

The AAVP is working on the design for an AA system covering all of the SKA frequencies up to $\sim 1.4 \mathrm{GHz}$. As has been noted, this will be as two or more separate arrays tailored to match the technical requirements against the sky noise. The
$A_{\text {eff }}$ requirements against $T_{\text {sky }}$ is illustrated in Figure 1 . The expectation is that AA-Lo will be a sparse array to gain $A_{\text {eff }}$ with increasing $\lambda$ to combat sky noise, which may be at the expense of beam precision. At higher frequencies sky noise drops and the demands of the science experiments merits a close packed array, AA-hi, Nyquist sampling the incoming wavefront. A full discussion on the AA design can be found in the SKADS White Paper (Faulkner et al. 2010).

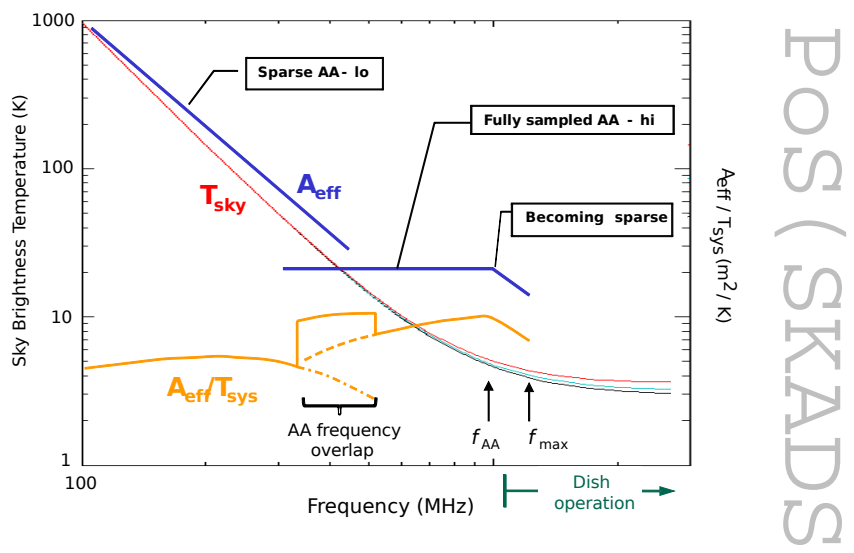

Fig. 1: Illustrative performance requirements considering Sky noise of the aperture arrays as part of the overall AA system

The AAVP main deliverables for the aperture arrays at the end of the PrepSKA period will be:

1. An approved, verified and costed design for Phase-1 AA integrated into the SKA Phase-1 system.

2. Clear development path to SKA Phase-2 AA system.

3. A design provided with initial 'Request for Quotation' documents with substantial technical detail for industrial competitive selection and delivery of pre-production parts. These parts are expected to be integrated within 2 years after funding availability (assumed coincident with PrepSKA end).

4. Design performance verification in the form of independently refereed documentation.

\section{SKA, phased arrays and key issues to investigate}

The organisation and work package structure for the AAVP is shown in Figure 2.

There is a coordination group, led by the Coordinator. This group reports to the full ESKAC on a regular, biannual, basis. Leaders of the AAVP Work Packages, WPs, work with the coordination group to guide the developments of WPs.

The technical programme is split into six WPs each with a leader and with one work package focusing on the technical development work for AAs. For reasons of optimization and 
consistency, the "system engineer" also coordinates AA-SDS and the "program engineer" coordinates AA-Tech.

Overall the aperture array developments are based on the same generic, parameterised design such that the cost/performance/power of the SKA Phase-1 and Phase-2 implementations can be optimised for the scientific return with the available technology. As many as possible of the sub-system developments will be reused between the demonstrator implementations. All the developments will use the knowledge gained within SKADS and have ongoing use and testing of EMBRACE and 2-PAD.

In short the six work packages consist of:

1. AA-SDS: AA system design studies, including simulations and costing.

2. AA-Tech: National coordinated tasks providing technology for AAs.

3. AA-Hi/ A3IV: Demonstrator: Aperture Array Astronomical Imaging Verification.

4. AA-Hi/ DAAVS: Demonstrator: Next generation Alldigital AA verification system.

5. AA-Lo: Aperture Array developments for the low frequency range.

6. AA-SEM: Aperture Array Site Emulator, the site infrastructure.

Detailed descriptions are given in the AAVP-Description of Work as a separate AAVP document.

Specifically, these are described for the Tasks constituting a Package.

Note that AA-Hi may consist of two demonstrators to demonstrate the full capability of AA-Hi in the frequency range form 400-1400 MHz. It is likely that the work will become more integrated at a later stage.

Note also that AA-Lo (intended for 70-450 MHz) has two package leaders. One development lead by INAF will focus on a next step using the LOFAR dual antenna approach while ICRAR will focus on a more exploratory track to be defined in more detail maximizing benefits from the MWA.

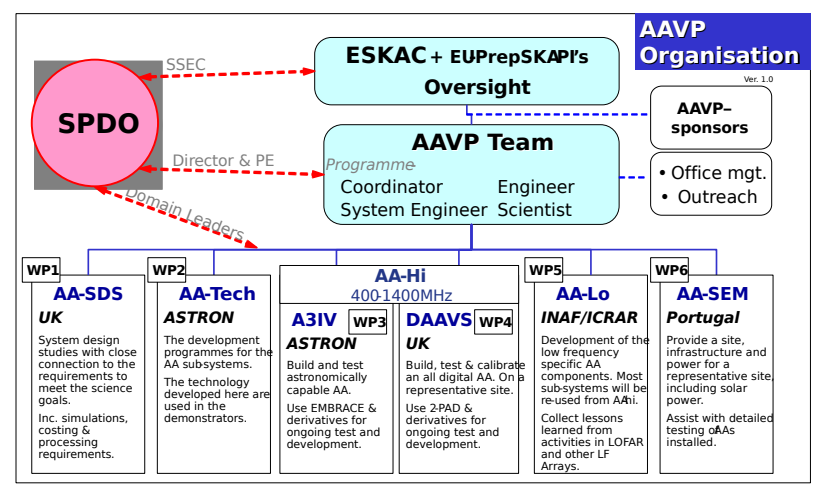

Fig. 2: AAVP Organisation including six Program Packages (described in the text) with proposed Package leaders. Detailed descriptions are given in the AAVP-Description of Work as a separate AAVP document.
Both the system package (SDS) and the AA-Tech are aligned with the overall SKA planning and in a sense are the "output" directed packages directly contributing to the SKA design.

As a result, all activities entail an "SKA design scope" i.e. an SKA design toward Phase 1 mass production.

\section{AAVP, some key issues}

The AAVP will be tackling all the issues of aperture array development, these are expected to be solvable and essentially requiring "only" competent organizational and engineering efforts to implement. There are, however, a few critical issues which have not yet been fully resolved in any development programme; these will be focussed upon by the AAVP:

- $T_{\text {sys }}$ : obtaining a low system temperature is essential to make the high performance AAs affordable. While this is true for all the SKA collector technologies, AA's are not cryogenically cooled and the noise behaviour is characterized by interaction between the (dense) array and the element LNA's. Hence, the principal components in determin$\operatorname{ing} T_{\text {sys }}$ are the elements, LNA, matching circuits and physical implementation. There is a specific Task, to concentrate on this issue and to conclude on the published models and approaches that have developed so far.

- Imaging: this is primarily maximising the dynamic range that can be achieved for well calibrated imaging. Again a challenge for all collector technologies, it will need to be demonstrated by the demonstrators and predicted for larger arrays in simulation. There are some inherent benefits with AAs: exquisite calibration capability, large unblocked aperture and inherent physical stability. However, the whole system will need to be designed and implemented well, since any imperfections will be exposed with these tests.

- Design: AAVP will resolve the issues of optimal transition frequencies of sparse toward dense arrays using higher frequency in AA-Lo as well as the optimal Nyquist frequency for AA-Hi.

- Cost: The AAs will use a lot of electronics, mechanics and interconnections, these could add up to an unacceptable cost, so this will be closely monitored and predicted using the cost tool developed in SKADS and now used by the International Project. AAVP through WP1 will coordinate the cost reduction work with required design performance to achieve the science requirements using all available experience including from SKADS.

It is worth noting that the low frequency arrays being implemented, LOFAR, MWA etc. will be commissioned over the next few years and will represent an important test bed for the performance of AAs in the SKA, many of the challenges are similar: varying beam shape with scan angle, multiple beams, wide field of view etc.

\section{Conclusion}

These conference proceedings on AAVP is only an introduction to the AAVP_ DOW now in final preparation. It is expected to 
be ready for release after the SKADS white paper in early 2010 and will contain all the activities and package/task details.

A period of new exciting developments is ahead of us again, requiring our full focus and dedication for a successful conclusion in preparation for SKA Phase 1.

\section{References}

AAVP website: www. SKA-AAVP . eu

secretary@SKA-AAVP . eu

Faulkner, A.J., et al., 2010, "SKADS White Paper"

Popping, A., \& Braun, R., 2008, A\&A 479, 903 